\title{
Thermodynamic Analysis of Gravitational Field Equations in Lyra Manifold
}

\author{
H. Moradpour, N. Sadeghnezhad, S. Ghaffari, and A. Jahan \\ Research Institute for Astronomy and Astrophysics of Maragha (RIAAM), P.O. Box 55134-441, Maragha, Iran \\ Correspondence should be addressed to H. Moradpour; hn.moradpour@gmail.com
}

Received 26 April 2017; Revised 18 June 2017; Accepted 16 July 2017; Published 14 August 2017

Academic Editor: Elias C. Vagenas

Copyright (c) 2017 H. Moradpour et al. This is an open access article distributed under the Creative Commons Attribution License, which permits unrestricted use, distribution, and reproduction in any medium, provided the original work is properly cited. The publication of this article was funded by $\mathrm{SCOAP}^{3}$.

\begin{abstract}
Considering the Einstein field equations in Lyra manifold and applying the unified first law of thermodynamics as well as the Clausius relation to the apparent horizon of FRW universe, we find the entropy of apparent horizon in Lyra manifold. In addition, the validity of second law of thermodynamics and its generalized form are also studied. Finally, we use the first law of thermodynamics in order to find the horizon entropy of static spherically symmetric spacetimes. Some results of considering (anti)de-Sitter and Schwarzschild metrics have also been addressed.
\end{abstract}

\section{Introduction}

Inasmuch as some observational data are not suitably described by the general relativity theory, physicists try to get gravitational theories in more agreement with nature [1-5]. The Riemannian geometry is the backbone of most of these theories, and it has been shown that the system horizon (as the boundary of system) has entropy, and indeed, there is a deep connection between the thermodynamics laws and the gravitational field equations [6-33]. This mutual relation between gravity and thermodynamics may help us in providing a description for the primary inflationary era as well as the current accelerating phase of universe [34]. In addition, due to such relation, geometry and the universe material content may approach a thermodynamic equilibrium [35].

In fact, the dark energy candidates, as the source of the current phase of universe, may modify the horizon entropy and thus its thermodynamics in both gravitational and cosmological setups [36-44]. Moreover, it seems that such modifications to the thermodynamics of system are in line with probable nonextensive thermodynamic properties of spacetime and the current universe [45-47].

A generalization of the Riemannian manifold has been introduced by Lyra [48] which modifies the Einstein field equations [49]. Changing Riemannian manifold by the Lyra geometry, one can also find a new scalar-tensor gravity in which both the tensor and scalar fields have geometrical interpretations [50]. Various aspects of the Einstein field equations in the Lyra manifold have been studied [51-61]. Using the Einstein field equations in Lyra manifold, Karade and Borikar have studied thermodynamic equilibrium of a spherical gravitating fluid [62].

Here, using the Einstein field equations in Lyra manifold, we are going to study the mutual relation between the field equations and the thermodynamics laws. Moreover, we are interested in obtaining the effects of Lyra generalization of Riemannian manifold on some thermodynamic properties of gravitating systems. In order to achieve this goal, applying the thermodynamics laws to the apparent horizon of FRW universe, we find an expression for the horizon entropy in Section 2. In addition, the validity of second and generalized second laws of thermodynamics will also be investigated in the next section. In Section 3, we use the Einstein field equations in Lyra manifold as well as the unified first law of thermodynamics in order to obtain the generalization of the Misner-Sharp mass [63] in this theory. Moreover, we use the obtained mass relation, field equations, and the first law of thermodynamics to find the entropy of static spherically symmetric horizons. A summary is presented in Section 4 . 
For the sake of simplicity, we consider $8 \pi G=c=\hbar=1$ in our study.

\section{Thermodynamics of the Apparent Horizon in Lyra Manifold}

In the Lyra manifold, the Einstein field equations are written as

$$
G_{\mu \nu}+\frac{3}{2} \phi_{\mu} \phi_{\nu}-\frac{3}{4} \phi_{\alpha} \phi^{\alpha} g_{\mu \nu}=T_{\mu \nu}
$$

where $\phi_{\nu}=g_{\mu \nu} \phi^{\mu}$ denotes the displacement vector field of Lyra manifold, and we set Einstein gravitational coupling constant to one $[48,54]$. As it is obvious, due to the geometrical field $\phi_{\mu}$, the Einstein gravitational field equations in Lyra and Riemannian manifolds differ from each other. Here, we are interested in investigating the effects of the displacement vector field on the apparent horizon entropy of the FRW universe and thus its thermodynamics.

In the cosmological setup, there are two common cases for $\phi_{\nu}$ vector including $\phi_{v}=(\beta(t), 0,0,0)$ and $\phi_{\nu}=(\alpha, 0,0,0)$, where $\alpha$ is constant, which help us in describing the current accelerating universe $[51,52]$. Here, we focus on the general case of $\phi_{v}=(\beta(t), 0,0,0)$ and find the entropy of apparent horizon.

The FRW universe is described by

$$
d s^{2}=-d t^{2}+a^{2}(t)\left[\frac{d r^{2}}{1-k r^{2}}+r^{2} d \Omega^{2}\right],
$$

where $a(t)$ is scale factor. In addition, $k=1,0,-1$ denotes the curvature parameter corresponding to closed, flat, and open universes, respectively. Therefore, $\phi_{\nu} \phi^{\nu}=\phi_{0} \phi^{0}=-\beta^{2}(t)$, and if universe is filled by an energy-momentum source as $T_{\mu}^{\nu}=$ $\operatorname{diag}(-\rho, p, p, p)$, then by defining the Hubble parameter as $H \equiv \dot{a} / a$, one obtains

$$
\begin{aligned}
& \rho=3\left[H^{2}+\frac{k}{a^{2}}\right]+\frac{3}{4} \beta^{2}(t), \\
& p=-3 H^{2}-2 \dot{H}-\frac{k}{a^{2}}+\frac{3}{4} \beta^{2}(t),
\end{aligned}
$$

for the Friedmann equations in the Lyra geometry. Here, $\rho$ and $p$ are the energy density and pressure of the isotropic fluid which supports the geometry, respectively. Moreover, here and from here onwards, dot denotes the derivative with respect to time. One can also use the Friedmann equations in order to find

$$
\dot{H}-\frac{k}{a^{2}}=-\frac{\rho+p}{2}+\frac{3}{4} \beta^{2}(t)
$$

In addition, since the apparent horizon of FRW universe is located at

$$
\tilde{r}_{A}=a(t) r_{A}=\frac{1}{\sqrt{H^{2}+\kappa / a(t)^{2}}},
$$

we can rewrite (4) in the form of

$$
\rho+p=\frac{2 \dot{\widetilde{r}}_{A}}{H \widetilde{r}_{A}^{3}}+\frac{6}{4} \beta^{2}(t) .
$$

In order to obtain continuity equation in our setup, one can define an energy-momentum tensor corresponding to the displacement vector field $\phi_{\mu}$ as

$$
T_{\mu}^{\nu}(\phi)=\frac{3}{4} \phi_{\alpha} \phi^{\alpha} \delta_{\mu}^{\nu}-\frac{3}{2} \phi_{\mu} \phi^{\nu}
$$

leading to

$$
T_{\mu}^{\nu}(\phi)=\operatorname{diag}(-\rho(\phi), p(\phi), p(\phi), p(\phi)),
$$

where $\rho(\phi)=p(\phi)=-(3 / 4) \beta^{2}(t)$ are the energy density and isotropic pressure corresponding to the displacement vector field of $\phi_{v}=(\beta(t), 0,0,0)$. If we define the state parameter corresponding to the Lyra displacement vector field as $w_{\phi} \equiv p(\phi) / \rho(\phi)$, then we have $w_{\phi}=1$ meaning that the displacement vector field acts as a stiff matter. Moreover, since $\rho(\phi)=-(3 / 4) \beta^{2}(t)$ is negative for real nonzero values of $\beta(t)$, the weak energy condition is not respected by a real nonzero $\phi_{\mu}$. In this manner, we have also $p(\phi)<0$ for $\beta(t) \neq 0$ meaning that the real Lyra displacement vector field induces a negative pressure into the background. This vector field may help us to build a model for an accelerating universe $[51,56]$ which can avoid the singularity, entropy, and horizon problems [53]. It is also worth mentioning that for imaginary Lyra displacement vector fields we have $\rho(\phi)=$ $p(\phi)>0$ meaning that the weak energy condition is satisfied. Therefore, since such fields do not lead to negative pressure, as the basic property of the accelerating universe, this kind of Lyra displacement vector field cannot be used to describe the inflationary and current phases of universe.

Let us define the total energy-momentum tensor $\left(\Theta_{\mu}^{v}\right)$ as $\Theta_{\mu}^{v} \equiv T_{\mu}^{\nu}+T_{\mu}^{\nu}(\phi)=\operatorname{diag}\left(-\rho_{e}, p_{e}, p_{e}, p_{e}\right)$ in which $\rho_{e}=\rho+\rho(\phi)$ and $p_{e}=p+p(\phi)$. In this manner, if $\rho \geq-\rho(\phi)=(3 / 4) \beta^{2}(t)$, then the $\rho_{e} \geq 0$ condition is respected. Moreover, rewriting the Einstein field equations in Lyra manifold as $G_{\mu}^{\nu}=\Theta_{\mu}^{\nu}$, one can easily obtain that the $\rho_{e} \geq 0$ condition is satisfied whenever the Hubble parameter meets the $H^{2} \geq 0$ condition, or equally $H$ should be a real quantity.

The Bianchi identity $\left(G_{\mu ; \nu}^{\nu}=0\right)$ implies $\Theta_{\mu ; \nu}^{\nu}=0$ which leads to

$$
\dot{\rho}+3 H(\rho+p)=\frac{3}{2}\left[\beta(t) \dot{\beta}(t)+3 H \beta^{2}(t)\right],
$$

for the continuity equation. This equation can also be decomposed into

$$
\begin{aligned}
& \dot{\rho}+3 H(\rho+p)=0, \\
& \dot{\beta}(t)+3 H \beta(t)=0,
\end{aligned}
$$

whenever there is no interaction between $\phi_{\mu}$ and the energymomentum source. 
2.1. The Entropy of Apparent Horizon. The Cai-Kim temperature of the apparent horizon and the projection of the fourdimensional energy-momentum tensor $T_{a}^{b}$ on the normal direction of the two-dimensional sphere with radius $\tilde{r}$ are as follows:

$$
\begin{aligned}
T & =\frac{1}{2 \pi \widetilde{r}_{A}}, \\
\psi_{a} & =T_{a}^{b} \partial_{b} \widetilde{r}+W \partial_{a} \widetilde{r},
\end{aligned}
$$

respectively $[20,21,64]$. Here, $W=(\rho-p) / 2$ is the work density. Additionally, the energy flux $\left(\delta Q^{m}\right)$ crossing the system boundary (the apparent horizon) is defined as $\delta Q^{m} \equiv$ $A \psi_{a} d x^{a}$, and therefore, simple calculations lead to

$$
d S_{A}=6 \pi \widetilde{r}_{A} V H(\rho+p) d t=8 \pi^{2} H \widetilde{r}_{A}^{4}(\rho+p) d t,
$$

where we used $T d S_{A} \equiv-\delta Q^{m}$ (the Clausius relation) and $V=$ $(4 \pi / 3) \widetilde{r}_{A}^{3}$ (aerial volume) relations to obtain this equation $[20,21,32,64]$. Now, inserting (6) into this equation and integrating the results, one can easily show that

$$
S_{A}=S_{B}+12 \pi^{2} \int \frac{H \beta^{2}(t)}{\left(H^{2}+k / a^{2}\right)^{2}} d t
$$

where $S_{B}=2 \pi A=8 \pi^{2} \widetilde{r}_{A}^{2}$ is the horizon entropy in the Einstein framework (or equally the Bekenstein entropy) if the Einstein field equations are written as $G^{\mu \nu}=T^{\mu \nu}$. Indeed, since $8 \pi G=\hbar=c=1$, we have $2 \pi=1 / 4 G$ and thus $S_{B}=$ $A / 4 G$ meaning that, at the $\beta(t) \rightarrow 0$ limit, the results of the Einstein theory are covered. Therefore, the second term on the RHS of this equation is the correction term to the horizon entropy due to the displacement vector filed of $\phi_{v}=(\beta(t)$, $0,0,0)$ considered in the Lyra geometry.

If one writes the Einstein field equations as $G^{\mu \nu}=8 \pi T^{\mu \nu}$, then simple calculations lead to $S_{A}=S_{B} / 8 \pi=A / 4$ for the horizon entropy. Moreover, it has also been shown that if the Einstein field equations are modified as $G^{\mu \nu}=8 \pi \Theta^{\mu \nu}$, where $\Theta^{\mu \nu}$ includes both the ordinary energy-momentum tensor $\left(T^{\mu \nu}\right)$ and correction terms $\left(\tau^{\mu \nu}\right)$, then one obtains

$$
S_{A}=\frac{A}{4}-8 \pi^{2} \int \frac{H\left(\rho_{c}+p_{c}\right)}{\left(H^{2}+k / a^{2}\right)^{2}} d t,
$$

for the horizon entropy [39-44]. Here, $\rho_{c}$ and $p_{c}$ denote the energy density and pressure components of $\tau^{\mu \nu}$, respectively. It is useful to mention here that, irrespective of a mutual interaction between various parts of $\Theta^{\mu \nu}$, the above result is available $[43,44]$. Therefore, one must expect that replacing $A / 4$ by $S_{B}$ in (16), we should get (15). As we have previously shown, in our case $\rho_{c}(\phi)=p_{c}(\phi)=-(3 / 4) \beta^{2}(t)$. Thus, replacing $A / 4$ by $S_{B}$ and inserting $\rho_{c}(\phi)=p_{c}(\phi)=-(3 /$ 4) $\beta^{2}(t)$ into (16), one can easily obtain (15) for the horizon entropy.

Finally, it is worthwhile mentioning that since we have not used (10) and (11) to obtain (15), this equation is valid irrespective of existence or absence of an interaction between Lyra displacement vector field and the energy-momentum source. This is in agreement with the properties of (16).
2.2. The Second and Generalized Second Laws of Thermodynamics. From (14), it is obvious that, independent of $\phi_{\mu}$, the second law of thermodynamics, stated as $d S_{A} / d t \geq 0$ [6], is met if the energy-momentum tensor satisfies the $\rho+p \geq 0$ condition. Therefore, if we define the state parameter as $w=$ $p / \rho$, then the $\rho+p \geq 0$ condition is covered for sources of positive energy density $(\rho \geq 0)$ only if we have $w \geq-1$.

Moreover, the generalized second law of thermodynamics (GSLT) states the total entropy of system $\left(S_{T}\right)$, including the horizon entropy $\left(S_{A}\right)$ and the entropy of fluid $\left(S_{m}\right)$ which supports the geometry, should not decrease or equally it has to respect the $d S_{T} / d t=d S_{A} / d t+d S_{m} / d t \geq 0$ condition $[7,8]$. In order to find $d S_{m}$ term, we use the first law of thermodynamics [65]

$$
T_{m} d S_{m}=d E+p d V
$$

where $T_{m}$ is the temperature corresponding to the supporter fluid. Inserting the $E=\rho V$ relation into (17), one finds that

$$
\frac{d S_{m}}{d t}=2 \pi \widetilde{r}_{A}[(\rho+p) \dot{V}+V \dot{\rho}]
$$

where we also used the $T_{m}=T=1 / 2 \pi \widetilde{r}_{A}$ relation [35]. Now, for noninteracting case, using (10) and (14) and the above result, we obtain

$$
\frac{d S_{T}}{d t}=\frac{d S_{A}}{d t}+\frac{d S_{m}}{d t}=6 \pi V(\rho+p) \dot{\tilde{r}}_{A},
$$

for the total entropy changes. Therefore, for a noninteracting expanding universe in which $\dot{\widetilde{r}}_{A}>0$, GSLT is satisfied if the dominant fluid respects the $\rho+p \geq 0$ condition. It is also obvious that, for spacetimes in that $\dot{H}=0$, we have $\dot{\tilde{r}}_{A}=H k / a^{2}\left(\sqrt{H^{2}+k / a^{2}}\right)^{3}$ meaning that, only in the flat and closed universe, GSLT is satisfied for a fluid respecting the $\rho+p \geq 0$ condition. In addition, for an open universe, GSLT is met if we have $\rho+p \leq 0$. Moreover, considering an interacting universe in which (10) is not valid, we use the original form of the continuity equation (9) and follow the recipe which led to (19) in order to get

$$
\begin{aligned}
& \frac{d S_{T}}{d t} \\
& \quad=6 \pi \widetilde{r}_{A} V\left[\frac{\dot{\widetilde{r}}_{A}(\rho+p)}{\tilde{r}_{A}}+\frac{\beta(t)}{2}(\dot{\beta}(t)+3 H \beta(t))\right],
\end{aligned}
$$

for the total entropy. Therefore, GSLT is valid whenever we have $\dot{\widetilde{r}}_{A}(\rho+p) / \widetilde{r}_{A}+(\beta(t) / 2)(\dot{\beta}(t)+3 H \beta(t)) \geq 0$. It has been shown that a constant $\beta(t)$ may help us in providing a description for the current acceleration phase of the universe expansion and thus the cosmological constant [51]. Therefore, we now focus on the $\beta(t)=\beta=$ constant case, leading to

$$
\frac{d S_{T}}{d t}=6 \pi \widetilde{r}_{A} V\left[\frac{\dot{\vec{r}}_{A}(\rho+p)}{\widetilde{r}_{A}}+\frac{3 H \beta^{2}}{2}\right],
$$

meaning that GSLT is met when we have $\beta^{2} \geq\left(2 \widetilde{r}_{A}^{2}(\rho+\right.$ p)/3) $\left(\dot{H}-k / a^{2}\right)$ yielding $\beta^{2} \geq 0$ for a flat universe $(k=0)$ of constant Hubble parameter $(\dot{H}=0)$. In obtaining the latter result, we used the $\dot{\widetilde{r}}_{A}=H\left(\left(\left(k / a^{2}\right)-\dot{H}\right) /\left(\sqrt{H^{2}+k / a^{2}}\right)^{3}\right)$ relation. 


\section{Thermodynamics of the Static Horizon}

We consider a four-dimensional static, spherically symmetric spacetime with a horizon located at $r_{h}$ and described by the metric

$$
d s^{2}=-f(r) d t^{2}+\frac{d r^{2}}{f(r)}+r^{2} d \Omega^{2} .
$$

Inserting metric (22) in (1), the $(t t)$ and $(r r)$ components of the Einstein tensors can be written as

$$
G_{0}^{0}=G_{1}^{1}=\frac{1}{r^{2}}\left(r f^{\prime}(r)+f(r)-1\right),
$$

where prime denotes derivative with respect to $r$. We consider a perfect fluid with $T_{\mu}^{v}=\operatorname{diag}(-\rho, p, p, p)$ which fills the spacetime. Therefore, since both the Einstein and energymomentum tensors are diagonal, only one component of the Lyra displacement vector field may be nonzero and its other components should be zero. We consider a time-like Lyra displacement vector field as $\varphi_{\nu}=(\varphi, 0,0,0)$, where $\varphi$ can be a function of $r$ or a constant [61, 62], and insert (23) into (1) to obtain [62]

$$
\rho=\frac{1}{r^{2}}\left(1-r f^{\prime}(r)-f(r)\right)-\frac{3}{2} \varphi_{0} \varphi^{0}+\frac{3}{4} \varphi_{\alpha} \varphi^{\alpha} .
$$

One can use (13) as well as the $d E \equiv A \psi_{a} d x^{a}$ relation in order to obtain [33]

$$
d E=4 \pi r^{2} \rho d r
$$

which leads to

$$
\begin{aligned}
d E & =4 \pi\left(1-r f^{\prime}(r)-f(r)-\frac{3}{2} r^{2} \varphi_{0} \varphi^{0}\right. \\
& \left.+\frac{3}{4} r^{2} \varphi_{\alpha} \varphi^{\alpha}\right) d r,
\end{aligned}
$$

and thus

$$
d E=4 \pi\left(1-\frac{d(r f(r))}{d r}+\frac{3 r^{2}}{4 f(r)} \varphi^{2}\right) d r
$$

where we also used the $\varphi_{\alpha} \varphi^{\alpha}=\varphi_{0} \varphi^{0}=-(1 / f(r)) \varphi^{2}$ relation to find the above result. We finally obtain

$$
E=4 \pi r(1-f(r))+3 \pi \int \frac{\varphi^{2} r^{2}}{f(r)} d r
$$

for the Misner-Sharp mass confined to radius $r$ in this theory. Therefore, for total mass of a black hole, we get $E=4 \pi r_{h}+$ $3 \pi \int_{0}^{r_{h}} \varphi^{2} r^{2} / f(r) d r$ covering the Einstein result $(E=4 \pi r)$ in the appropriate limit of $\varphi \rightarrow 0[11,63]$. Although we obtained $E=4 \pi r$ in the $\varphi \rightarrow 0$ limit, since we set the Einstein constant to one $(8 \pi G=1)$, we have $4 \pi=1 / 2 G$ and thus $E=r / 2 G$ in agreement with previous studies [11,33, 63]. Substituting (23) into (1), one easily finds that

$$
p=\frac{1}{r^{2}}\left(r f^{\prime}(r)+f(r)-1\right)+\frac{3}{4 f(r)} \varphi^{2},
$$

which finally leads to

$$
p d V=4 \pi\left(r f^{\prime}(r)+f(r)-1+\frac{3 r^{2}}{4 f(r)} \varphi^{2}\right) d r,
$$

where we used $d V=4 \pi r^{2} d r$. From (28) and (30), one gets $d E / d r_{h}=4 \pi+3 \pi \operatorname{limit}\left(\varphi^{2} r^{2} / f(r)\right)_{r \rightarrow r_{h}}$ and $p\left(d V / d r_{h}\right)=$ $4 \pi\left(r_{h} f^{\prime}\left(r_{h}\right)-1+\operatorname{limit}\left(\left(3 r^{2} / 4 f(r)\right) \varphi^{2}\right)_{r \rightarrow r_{h}}\right)$, respectively. It means that, in order to have meaningful expressions for $d E / d r_{h}$ and $p\left(d V / d r_{h}\right)$, the limit $\left(\varphi^{2} r^{2} / f(r)\right)_{r \rightarrow r_{h}}$ term should not diverge. Now, using these results as well as the first law of thermodynamics $\left(T d S_{A}=p d V+d E\right)$, we obtain

$$
T \frac{d S_{A}}{d r_{h}}=6 \pi \operatorname{limit}\left(\frac{\varphi^{2} r^{2}}{f(r)}\right)_{r \rightarrow r_{h}}+4 \pi r_{h} f^{\prime}\left(r_{h}\right),
$$

and therefore

$$
\begin{aligned}
& T \frac{d S_{A}}{d r_{h}} \\
& \quad=\frac{f^{\prime}\left(r_{h}\right)}{4 \pi}\left(\frac{d S_{B}}{d r_{h}}+24 \pi^{2} \operatorname{limit}\left(\frac{\varphi^{2} r^{2}}{f(r) f^{\prime}(r)}\right)_{r \rightarrow r_{h}}\right),
\end{aligned}
$$

where $S_{B}=2 \pi A=8 \pi^{2} r_{h}^{2}$ is the Bekenstein entropy. Again, since $8 \pi G=c=\hbar=1$, it is easy to obtain the familiar form of the Bekenstein entropy as $S_{B}=2 \pi A=A / 4 G$. Moreover, because we have $T \equiv f^{\prime}\left(r_{h}\right) / 4 \pi$, we finally obtain

$$
S_{A}=S_{B}+24 \pi^{2} \int \frac{\varphi^{2} r_{h}^{2}}{f\left(r_{h}\right) f^{\prime}\left(r_{h}\right)} d r_{h},
$$

for the horizon entropy, covering the Einstein result at the appropriate limit of $\varphi \rightarrow 0$. In order to have a well-defined entropy, the second term of RHS of this equation should not be divergent meaning that, at the $r \rightarrow r_{h}$ limit, the zero-zero component of Lyra time-like displacement vector field should meet the $\varphi / f(r) \rightarrow \infty$ condition.

Finally, let us study the case of $\varphi=l \sqrt{f(r)}$, where $l$ is arbitrary. Since metric is a spherically symmetric static metric and thus the Einstein tensor is static, for spherically symmetric static energy-momentum tensors, $l$ may be a constant or a function of $r$ and therefore

$$
S_{A}=S_{B}+24 \pi^{2} \int \frac{l^{2} r_{h}^{2}}{f^{\prime}\left(r_{h}\right)} d r_{h}
$$

leading to

$$
\begin{aligned}
& S_{A}^{d}=2 \pi A\left(1-\frac{3 l^{2}}{4 \Lambda}\right), \\
& S_{A}^{c}=2 \pi A\left[1+\frac{3 l^{2} A^{3 / 2}}{80 m \pi^{3 / 2}}\right],
\end{aligned}
$$

for $f(r)=1-\Lambda r^{2}$ and $f(r)=1-2 m / r$ (Schwarzschild), respectively. In obtaining the above results, we assumed that $l$ is constant. It is obvious that, for real values of $l, S_{A}^{c}>0$ and we have $S_{A}^{d} \geq 0$ if and only if $3 l^{2} \leq 4 \Lambda$. In addition, it is also easy to verify that, for real values of $l$, entropy is always positive and nonzero for an anti-de-Sitter universe, where $\Lambda<0$. 


\section{Summary}

Throughout this work, applying the unified first law of thermodynamics to the apparent horizon of FRW universe and using the Clausius relation as well as the Cai-Kim temperature, we could obtain a relation for the effects of Lyra displacement vector on the entropy of apparent horizon. The validity of second and generalized second laws of thermodynamics has also been studied.

Moreover, considering the Einstein field equations in the Lyra manifold for a spherically symmetric static metric together with the first law of thermodynamics, an expression for the entropy of the static event horizon has also been obtained. Finally, the horizon entropy for (anti)de-Sitter and Schwarzschild black holes in the Lyra manifold was also investigated.

\section{Conflicts of Interest}

The authors declare that there are no conflicts of interest regarding the publication of this paper.

\section{Acknowledgments}

The work of H. Moradpour has been supported financially by Research Institute for Astronomy \& Astrophysics of Maragha (RIAAM) under Project no. 1/4717-170.

\section{References}

[1] S. Nojiri and S. D. Odintsov, "The new form of the equation of state for dark energy fluid and accelerating universe," Physics Letters B, vol. 639, no. 3-4, pp. 144-150, 2006.

[2] K. Bamba, S. Capozziello, S. Nojiri, and S. D. Odintsov, "Dark energy cosmology: the equivalent description via different theoretical models and cosmography tests," Astrophysics and Space Science, vol. 342, no. 1, pp. 155-228, 2012.

[3] S. Nojiri and S. D. Odintsov, "Unified cosmic history in modified gravity: from $F(R)$ theory to Lorentz non-invariant models," Physics Reports, vol. 505, no. 2-4, p. 59, 2011.

[4] S. Capozziello and V. Faraoni, Beyond Einstein Gravity, Springer, Berlin, Germany, 2010.

[5] F. S. N. Lobo, "Beyond Einstein's general relativity," Journal of Physics: Conference Series, vol. 600, no. 1, Article ID 012006, 2015.

[6] S. W. Hawking, "Gravitational radiation from colliding black holes," Physical Review Letters, vol. 26, p. 1344, 1971.

[7] J. D. Bekenstein, "Black holes and entropy," Physical Review. D. Particles and Fields. Third Series, vol. 7, pp. 2333-2346, 1973.

[8] J. D. Bekenstein, "Generalized second law of thermodynamics in black-hole physics," Physical Review D, vol. 9, no. 12, pp. 32923300, 1974.

[9] T. Jacobson, "Thermodynamics of spacetime: the Einstein equation of state," Physical Review Letters, vol. 75, p. 1260, 1995.

[10] C. Eling, R. Guedens, and T. Jacobson, "Nonequilibrium thermodynamics of spacetime," Physical Review Letters, vol. 96, no. 12, Article ID 121301, 2006.

[11] A. Paranjape, S. Sarkar, and T. Padmanabhan, "Thermodynamic route to field equations in Lanczos-LOVelock gravity," Physical Review D, vol. 74, no. 10, Article ID 104015, 2006.
[12] H. Maeda, "Final fate of spherically symmetric gravitational collapse of a dust cloud in Einstein-Gauss-Bonnet gravity," Physical Review D, vol. 73, no. 10, Article ID 104004, 17 pages, 2006.

[13] H. Maeda and M. Nozawa, "Generalized Misner-Sharp quasilocal mass in Einstein-Gauss-Bonnet gravity," Physical Review D, vol. 77, no. 6, Article ID 064031, 13 pages, 2008.

[14] R. G. Cai, L. M. Caoand, and N. Ohta, "Topological black holes in Horava-Lifshitz gravity," Physical Review D, vol. 80, no. 2, Article ID 024003, 2009.

[15] T. Padmanabhan, "Thermodynamical aspects of gravity: new insights," Reports on Progress in Physics, vol. 73, Article ID 046901, 2010.

[16] T. Padmanabhan, "Equipartition of energy in the horizon degrees of freedom and the emergence of gravity," Modern Physics Letters A: Particles and Fields, Gravitation, Cosmology, Nuclear Physics, vol. 25, no. 14, pp. 1129-1136, 2010.

[17] T. Padmanabhan, "Surface density of spacetime degrees of freedom from equipartition law in theories of gravity," Physical Review D, vol. 81, Article ID 124040, 2010.

[18] M. Akbar and R.-G. Cai, "Thermodynamic behavior of field equations for $f(R)$ gravity," Physics Letters $B$, vol. 648, no. 2-3, pp. 243-248, 2007.

[19] M. Akbar and R. G. Cai, "Friedmann equations of FRW universe in scalar-tensor gravity, $f(R)$ gravity and first law of thermodynamics," Physics Letters B, vol. 635, no. 1, pp. 7-10, 2006.

[20] M. Akbar and R. G. Cai, "Thermodynamic behavior of the Friedmann equation at the apparent horizon of the FRW universe," Physical Review D, vol. 75, Article ID 084003, 2007.

[21] R. G. Cai and S. P. Kim, "First law of thermodynamics and Friedmann equations of Friedmann-Robertson-Walker universe," Journal of High Energy Physics, vol. 0502, p. 050, 2005.

[22] R.-G. Cai and L.-M. Cao, "Unified first law and the thermodynamics of the apparent horizon in the FRW universe," Physical Review D: Particles, Fields, Gravitation, and Cosmology, vol. 75, no. 6, Article ID 064008, 11 pages, 2007.

[23] R. G. Cai and L. M. Cao, "Thermodynamics of apparent horizon in brane world scenario," Nuclear Physics B, vol. 785, no. 1-2, pp. 135-148, 2007.

[24] A. Sheykhi, B. Wang, and R.-G. Cai, "Thermodynamical properties of apparent horizon in warped DGP braneworld," Nuclear Physics B, vol. 779, no. 1-2, pp. 1-12, 2007.

[25] A. Sheykhi, B. Wang, and R.-G. Cai, "Deep connection between thermodynamics and gravity in Gauss-Bonnet braneworlds," Physical Review D, vol. 76, no. 2, Article ID 023515, 5 pages, 2007.

[26] A. Sheykhi, "Thermodynamical interpretation of gravity in braneworld scenarios," Journal of Cosmology and Astroparticle Physics, vol. 05, p. 019, 2009.

[27] A. Sheykhi, "Thermodynamics of apparent horizon and modified Friedman equations," The European Physical Journal C, vol. 69 , p. $265,2010$.

[28] A. Sheykhi, “Thermodynamics of interacting holographic dark energy with the apparent horizon as an IR cutoff," Classical and Quantum Gravity, vol. 27, no. 2, Article ID 025007, 2010.

[29] R. G. Cai and N. Ohta, "Horizon thermodynamics and gravitational field equations in Hořava-Lifshitz gravity," Physical Review D, vol. 81, Article ID 084061, 2010.

[30] A. Sheykhi, "Thermodynamics of the apparent horizon in infrared modified Horava-Lifshitz gravity," Physical Review D, vol. 87, Article ID 024022, 2013. 
[31] A. Sheykhi, M. H. Dehghani, and R. Dehghani, "Horizon thermodynamics and gravitational field equations in quasitopological gravity," General Relativity and Gravitation, vol. 46, no. 4, pp. 1-14, 2014.

[32] H. Moradpour, "Thermodynamics of flat FLRW universe in Rastall theory," Physics Letters B, vol. 757, pp. 187-191, 2016.

[33] H. Moradpour and G. I. Salako, “Thermodynamic analysis of the static spherically symmetric field equations in rastall theory," Advances in High Energy Physics, vol. 2016, Article ID 3492796, 5 pages, 2016.

[34] H. Moradpour, R. C. Nunes, E. M. Abreu, and J. A. Neto, "A note on the relations between thermodynamics, energy definitions and Friedmann equations," Modern Physics Letters A. Particles and Fields, Gravitation, Cosmology, Nuclear Physics, vol. 32, no. 13, 1750078, 20 pages, 2017.

[35] J. P. Mimoso and D. Pavón, "Considerations on the thermal equilibrium between matter and the cosmic horizon," Physical Review D, vol. 94, Article ID 103507, 2016.

[36] C. J. Feng, X. Z. Li, and X. Y. Shen, "Thermodynamic of the ghost dark energy universe," Modern Physics Letters A, vol. 27, Article ID 1250182, 2012.

[37] A. Sheykhi, "Entropy and thermodynamics of ghost dark energy," Canadian Journal of Physics, vol. 92, no. 6, pp. 529-532, 2014.

[38] H. Moradpour, M. T. Mohammadi Sabet, and A. Ghasemi, "Thermodynamic analysis of universes with the initial and final de Sitter eras," Modern Physics Letters A, vol. 30, no. 31, Article ID 1550158, 2015.

[39] H. Ebadi and H. Moradpour, "Thermodynamics of universe with a varying dark energy component," International Journal of Modern Physics D, vol. 24, no. 14, Article ID 1550098, 2015.

[40] S. Mitra, S. Saha, and S. Chakraborty, "Universal thermodynamics in different gravity theories: conditions for generalized second law of thermodynamics and thermodynamical equilibrium on the horizons," Annals of Physics, vol. 355, pp. 1-20, 2015.

[41] S. Mitra, S. Saha, and S. Chakraborty, "A study of universal thermodynamics in Lanczos-LOVelock gravity," General Relativity and Gravitation, vol. 47, no. 6, Art. 69, 18 pages, 2015.

[42] S. Mitra, S. Saha, and S. Chakraborty, "A study of universal thermodynamics in brane world scenario," Advances in High Energy Physics, vol. 2015, Article ID 430764, 9 pages, 2015.

[43] H. Moradpour and M. T. Mohammadi Sabet, "Thermodynamic descriptions of polytropic gas and its viscous type as dark energy candidates," Canadian Journal of Physics, vol. 94, no. 3, pp. 334341, 2016.

[44] H. Moradpour and R. Dehghani, “Thermodynamical study of FRW universe in quasi-topological theory," Advances in High Energy Physics, vol. 2016, Article ID 7248520, 10 pages, 2016.

[45] C. Tsallis and L. J. L. Cirto, "Black hole thermodynamical entropy," European Physical Journal C, vol. 73, no. 7, p. 2487, 2013.

[46] H. Moradpour, "Implications, consequences and interpretations of generalized entropy in the cosmological setups," International Journal of Theoretical Physics, vol. 55, no. 9, pp. 41764184, 2016.

[47] R. C. Nunes, E. M. Barboza Jr, E. M. C. Abreu, and J. A. Neto, "Probing the cosmological viability of non-gaussian statistics," Journal of Cosmology and Astroparticle Physics, vol. 08, p. 051, 2016.

[48] G. Lyra, "Über eine modifikation der riemannschen geometrie," Mathematische Zeitschrift, vol. 54, pp. 52-64, 1951.
[49] D. K. Sen, "A static cosmological model," Zeitschrift für Physik B Condensed Matter, vol. 149, p. 311, 1957.

[50] D. K. Sen and K. A. Dunn, "A scalar-tensor theory of gravitation in a modified Riemannian manifold," Journal of Mathematical Physics, vol. 12, p. 578, 1971.

[51] W. D. Halford, "Cosmological theory based on Lyra's geometry," Australian Journal of Physics, vol. 23, no. 5, p. 863, 1970.

[52] A. Beesham, "FLRW cosmological models in Lyra's manifold with time dependent displacement field," Australian Journal of Physics, vol. 41, no. 6, pp. 833-842, 1988.

[53] T. Singh and G. P. Singh, "Lyra's geometry and cosmology: a review," Fortschritte der Physik. Progress of Physics, vol. 41, no. 8, pp. 737-764, 1993.

[54] F. Darabi, Y. Heydarzade, and F. Hajkarim, "Stability of Einstein static universe over Lyra geometry," Canadian Journal of Physics, vol. 93, no. 12, pp. 1566-1570, 2015.

[55] M. Yavari, "The exact solutions of the raychaudhuri spacetime based on Lyra manifold," Georgian Electronic Scientific Journals: Physics, vol. 1, p. 9, 2013.

[56] H. H. Soleng, "Cosmologies based on Lyra's geometry," General Relativity and Gravitation, vol. 19, no. 12, pp. 1213-1216, 1987.

[57] G. S. Khadekar, Pradhan, and K. Srivastava, "Cosmological models in lyra geometry: kinematics tests," Letters in Mathematical Physics, 2005, https://arxiv.org/abs/gr-qc/0508099.

[58] M. Khurshudyan, "Interacting extended Chaplygin gas cosmology in Lyra manifold," Astrophysics and Space Science, vol. 360, p. 44, 2015.

[59] A. H. Ziaie, A. Ranjbar, and H. R. Sepangi, "Trapped surfaces and the nature of singularity in Lyra's geometry," Classical and Quantum Gravity, vol. 32, no. 2, Article ID 025010, 025010, 30 pages, 2015.

[60] M. U. Dogru and I. Yilmaz, "Expanding and nonexpanding conformal wormholes, in scalar-tensor theory," International Journal of Modern Physics. D. Gravitation, Astrophysics, Cosmology, vol. 24, no. 8, Article ID 1550064, 2015.

[61] M. Yavari, "Exact solutions of the static cylindrically symmetric metric based on Lyra geometry for the viscous fluid with massless scalar field," Bulletin of the Georgian National Academy of Sciences. New Series, vol. 10, no. 3, pp. 55-59, 2016.

[62] T. M. Karade and S. M. Borikar, "Thermodynamic equilibrium of a gravitating sphere in Lyra's geometry," General Relativity and Gravitation, vol. 9, no. 5, pp. 431-436, 1978.

[63] C. W. Misner and D. H. Sharp, "Relativistic equations for adiabatic, spherically symmetric gravitational collapse," Physical Review, vol. 136, no. 2, pp. B571-B576, 1964.

[64] R. G. Cai, L. M. Cao, and Y. P. Hu, "Hawking radiation of an apparent horizon in a FRW universe," Classical and Quantum Gravity, vol. 26, Article ID 155018, 2009.

[65] H. B. Callen, Thermodynamics and Introduction to Thermostatics, John Wiley and Sons, New York, NY, USA, 1985. 

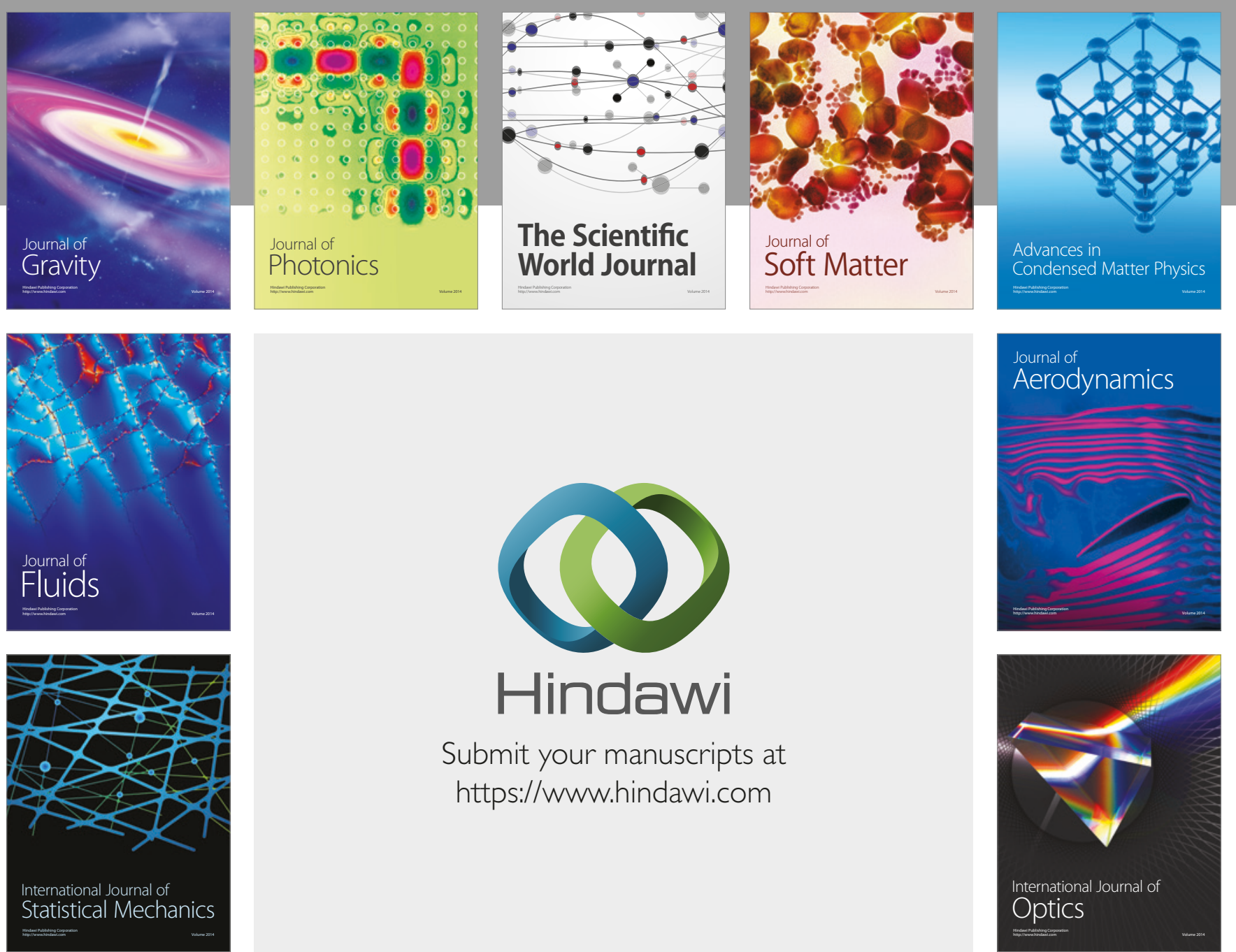

Submit your manuscripts at

https://www.hindawi.com
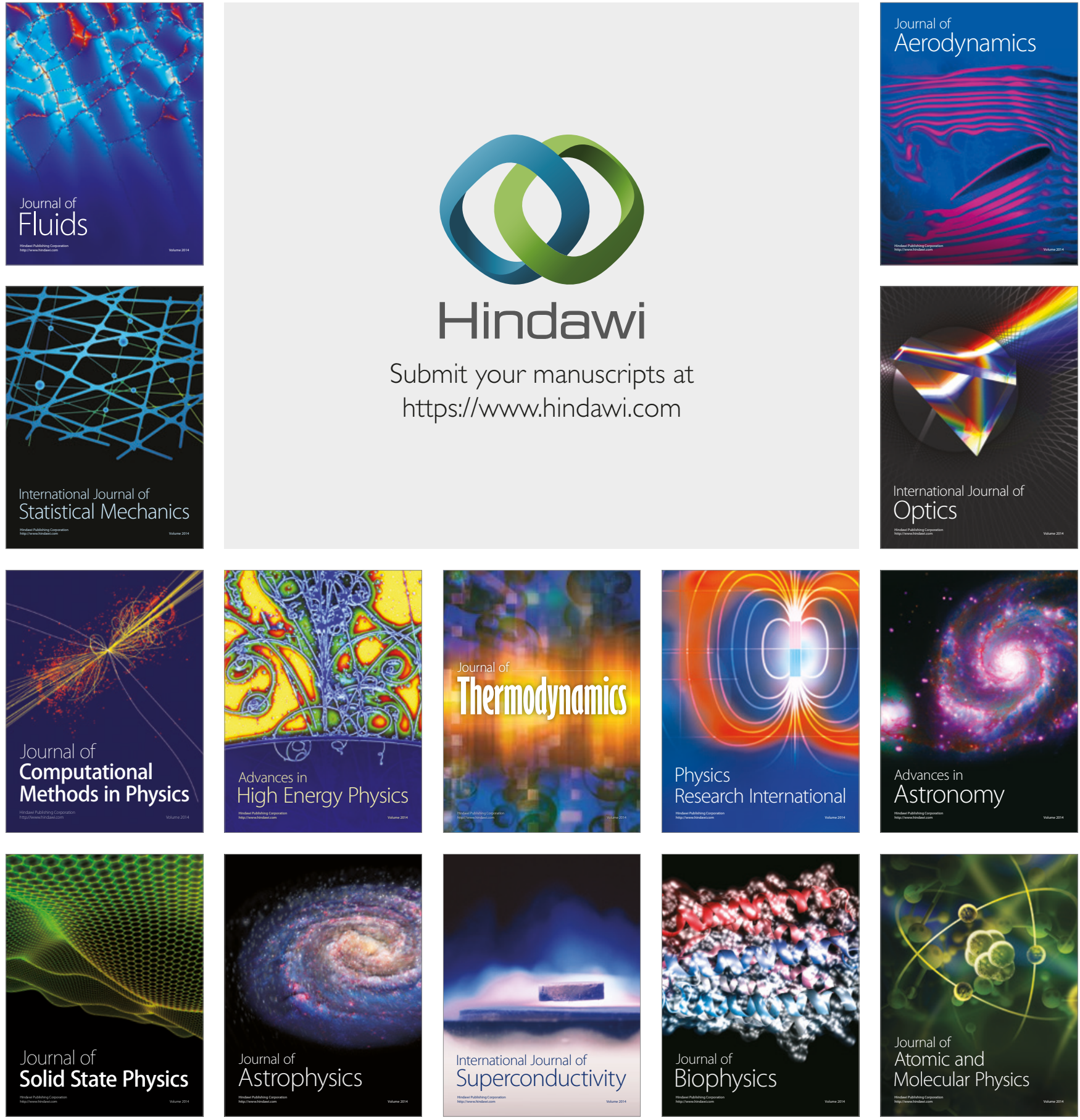